\title{
Research development of compound bioflocculant
}

\author{
Lixin $\mathrm{Li}^{1,2}$, Fang $\mathrm{Ma}^{1}$, Tong Pan ${ }^{2}$ \\ ${ }^{1}$ State Key Laboratory of Urban Water Resource and Environment, Harbin Institute of Technology, \\ Harbin, 150090, China
}

${ }^{2}$ School of Environment and Chemical Engineering, Heilongjiang University of Science and Technology, Harbin, 150022, China

Keywords: compound; bioflocculant; development.

\begin{abstract}
The research background and characteristics of compound bioflocculant (CBF) were introduced. The paper focused on research and application of CBF, including producing compound bioflocculant by Low-cost substrate and engineering application of CBF. Finally the research development of CBF in the future were discussed.
\end{abstract}

\section{Introduction}

Flocculants are useful agents in the aggregation of colloids, cells and suspended solids and are commonly used for drinking water production, waste water treatment, fermentation processes, and food production [1]. In general, flocculants are classified into three groups: inorganic flocculants, such as aluminum sulfate and polyaluminum chloride; organic synthetic flocculants, such as polyacrylamide derivatives and polyethylene imine; and naturally occurring flocculants, such as chitosan, sodium alginate, and microbial flocculants. Despite the effective flocculating performance and low cost of the synthetic chemical flocculants, their use has resulted in some health and environmental problems [2]. In contrast, bioflocculants, extracellular biopolymeric substances secreted by bacteria, fungi, algae and yeast are biodegradable and nontoxic flocculants [3, 4].

Microbial flocculants is a kind of metabolites produced by the microorganism, the main ingredients are glycoproteins, polysaccharides, protein and DNA et al. It is obtained by the fermentation of bacteria and fungi, extraction, purification in use of microbial technology, is a new type of water treatment agent [5-7]. Microbial flocculant is biodegradable, non-toxic and safe of high efficient for wastewater treatment. The study on the bioflocculant began in the 1950s, Japanese scholars first discovered microbial culture liquid with flocculation. In 1976, Nakamura et al studied microbes producing flocculation effect, and raised the research hotspot for microbial flocculant [8]. In 1986, Kurane et al [9] used Rhodococcus erythropolis S1strain isolated from nature to produce flocculant NOC-1with good flocculation effect. The research of microbial flocculant, has been mainly concentrated on producing strain screening and flocculant fermentation liquid for wastewater treatment in laboratory scale tests, there were many problems in the process of research, for example, high cost for producing bioflocculant, screening the strain was difficult, the flocculation process and mechanism is not clear, and so on. In recent years, in order to improve the effect of flocculation, reduce the producing bioflocculant cost, compound bioflocculant began to receive the much attention of researchers $[10,11]$.

Bioflocculants are mainly composed of macromolecular substances, such as polysaccharide and protein $[12,13]$. The composition and properties of bioflocculants depend on type of bioflocculantproducing microorganisms (BPMs), composition of media and environmental conditions [14]. Although so many studies about bioflocculants have been done, low flocculating capability and high cost are still the major problems in limiting bioflocculants development for practical application [15]. Consequently, screening for microorganisms producing bioflocculants with high flocculating activities [2, 4], improving the flocculating performance of the biofloccualnts [16], and seeking for low-cost substrates for producing bioflocculants have become recent hot topics in this field [17]. 
The concept of compound bioflocculant (CBF) was first put proposed by Professor Ma of Harbin Institute of Technology $[16,18]$. The raw fermentation material of compound bioflocculant was agricultural waste straw. The high-efficiency combined production of bioflocculant utilized the composition flora of cellulose degrading bacteria and flocculant producing bacteria, through two stage fermentation, realized the coupling of cellulose scarification section and producing flocculating section. Therefore, compound bioflocculant on the basis of compound bacteria has advantages of bacterial activity, the flocculation effect, the cost of production, compared with single microorganism producing flocculant, becomes the hot spot in the current research.

Ma et al. $[16,18]$ put forward the conception of CBF, which was produced by mixed culture of bioflocculant-producing microorganisms and could exhibit excellent flocculating ability. The compound bioflocculant in the present research was produced by mixed culture of two bioflocculant-producing microorganisms, Rhizobium radiobacter F2 and Bacillus sphaeicus F6. CBF possesses higher flocculating activity than bioflocculants by single culture of F2 and F6 [19].So far, the domestic scholars mainly concentrated in the screening and optimization of compound bacteria culture [20], application of compound bioflocculant for treatment raw water and wastewater. The domestic and international research status of compound bioflocculant in recent years and its future research development were introduced in the paper.

\section{The characteristics of the compound bioflocculant}

The compound bacteria theory as the foundation, the compound bioflocculant was produced mainly by mixed culture of more than two kinds of bacteria. Various strains promotes proliferation each other through synergistic action, and gave full play to their respective advantages, formed an effective complementary, so that the compound bioflocculant had maximum flocculating ability.

The compound bioflocculant was produced by two step fermentation, fermentation technology has the composite characteristics. Screening and construction of flocculant producing bacteria realized producing stable and high efficient bioflocculant; screening bacteria cellulose degradation bacteria and construction of bacteria flora realized the efficient and quick saccharification period. Two step fermentation by organic combination had efficient and simple process for producing the compound bioflocculant.

The main ingredients were including extracellular secretions of the producing flocculant strains, cellulose degrading bacteria bodies, flocculation bacteria bodies, cellulose residue etc.

\section{Producing compound bioflocculant by Low-cost substrate}

Compound bioflocculant has not been industrialized production and application of the large scale, one of the reasons is that the production cost is too high. So looking for cheap substrate and the development of low cost substrate is very necessary. The medium of cheap carbon source and nitrogen source for bioflocculant production can effectively solve the problem of high-cost culture medium, which offer potential opportunities for commercial production of CBF. Some scholars used dairy wastewater and sauce wastewater or other food wastewater as cheap culture medium to culture bioflocculant producing bacteria [21, 22]. It provided ideas of cheap substrate for producing compound bioflocculant. Chinese scholars in recent years has done a lot of researches in cheap substrate for the production of compound bioflocculant. Ma et al $[16,18]$ used cheap cellulose as a substrate of compound bioflocculant by two stage fermentation process for preparing CBF. Then Ma et al done a lot of researches in utilizing rice straw as substrate for preparation of compound bioflocculant [23]. Other Chinese scholars [24-27] used the distillery wastewater, brewery wastewater, food processing wastewater, sauce wastewater, monosodium glutamate wastewater to produce compound bioflocculant, then they achieved satisfying results, the studies laid the foundation for the industrial production of compound bioflocculant. 


\section{Application of compound bioflocculant in water treatment}

\section{Treatment of drinking water}

The compound bioflocculant achieved good results in surface water treatment. Such as: Ma ET all's [28] study showed that when CBF dosage was about $14 \mathrm{ml} / \mathrm{L}$, the $\mathrm{pH}$ value was 7.5 , the addition of $\mathrm{CaCl} 2$ was $1.5 \mathrm{ml} / \mathrm{L}$. The optimal flocculation effect was obtained in the treatment of Songhua River water. The temperature has little influence on the flocculation efficiency.

\section{Treatment of domestic sewage}

Domestic sewage with the characteristics of high organic content, complex composition of the water quality, but compound bioflocculant in treatment domestic sewage showed good treatment properties. When CBF was used for treatment of domestic sewage, all kinds of pollutant removal rate was above 60\% [29]; compound bioflocculant XZ has the high removal efficiency for SS and Chroma of domestic sewage, XZ also could improve the related indexes of activated sludge [10].

\section{Treatment of industrial wastewater}

Zhang Yuling et all's [30] research showed that when CBF treated starch wastewater, flocculation rate reached 80.0-90.3\%.By the compound bioflocculant XJBF-1 for treatment of starch wastewater, printing and dyeing wastewater and landfill leachate, COD removal rate reached $88 \%, 66 \%$ and $58 \%$ respectively, and the treatment effect of starch wastewater and dyeing wastewater is better than that of the action polyacrylamide [31].

\section{Conclusions}

The compound bioflocculant research at home and abroad had been developed for more than 10 years, a lot of achievements were obtained. But large-scale applications of CBF met a certain difficulty. Therefore, a single chemical or bioflocculant are not suited to development needs of the current water pollution control engineering technology, the urgent needs of the market should be efficient, environmental friendly composite flocculants products. Research and development of new key technology of composite biological flocculants industry, provided new flocculants products and technology support for the safety of drinking water supply, water pollution control and water deep purification treatment .it would be an important direction for future development of bioflocculant.

\section{Acknowledgments}

This work was supported by Grants from the National High Technology Research and Development Program of China (863 Program) (No. 2009AA062906); the 'Twelfth Five-Year Plan’National Key Technology R\&D Program of China (No. 2012BAD14B06-04); the National Natural Science Foundation of China (No. 51478140 and 51408200); Science and Technology Research Project of Heilongjiang Province Education Department (No.12541701) and Promising Youngsters Training Program of Heilongjiang University of Science and Technology (No. Q20120201).

\section{References}

[1] Shih, I.L.; Van, Y.T.; Yeh, L.C.; Lin, H.G.; Chang, Y.N., Production of a biopolymer flocculant from Bacillus licheniformis and its flocculation properties. Bioresour. Technol., 78, pp.267-272, 2001.

[2] Xia Si-Qing, Zhang Zhi-Qiang, Wang Xue-Jiang, Yang A-Ming, Chen Ling, Zhao Jian-Fu , Didier Leonard , Nicole Jaffrezic-Renault, Production and characterization of a bioflocculant by Proteus mirabilis TJ-1. Bioresour. Technol., 99,pp. 6520-6527,2008.

[3] Salehizadeh, H.; Shojaosadati, S.A., Extracellular biopolymeric flocculants: recent trends and biotechnological importance.Biotechnol. Adv., 19, pp.371-385, 2001.

[4] He Ning, Li Yin, Chen Jian, Production of a novel polygalacturonic acid bioflocculant REA-11 by Corynebacterium glutamicum.Bioresour, Technol., 94, pp. 99-105, 2004. 
[5] Li He-Ping,Zheng Ze-Geng,Zhu Zhu,Zheng Huai-Li, Microbial flocculant, Chongqing Environmental Science, 22, pp.18-21, 2000.

[6] Gan Guang-Feng, Gan Li, The progress of the researches on high polymeric flocculants, Industrial Water Treatment, 19, pp.6-7, 1999.

[7] J. Nakamura, Purification and chemical analysis of microbial cell flocculant produced by Aspergillus sojae AJ 7002. Agric. Bio. Chem., 40, pp.619-624, 1976.

[8] J. Nakamura, S. Miyashiro, Y. Hirose, Screening, isolation, and some properties of microbial cell flocculants, Agric. Bio. Chem., 40, pp.377-383, 1976.

[9] R. Kurane, T. Kazuki, T. Kiyoshi, Culture condition for production of microbial flocculant by Rhodococcus erythropolis, Agri. Bio. Chem., 50, pp.2309-2313, 1986.

[10] Fang Ming-Zhong, Studies on the production and flocculation characteristics of complex bioflocculant, Guangzhou:Guangdong University of Technology. 2008.

[11] Zhu Yan-bin,Ma Fang,Yang Ji-xian,Li Da-peng, Combination effect of flocculants for water treatment and development of compound flocculants, Journal of Harbin Institute of Technology , 42, pp.1254-1258, 2010.

[12] Lu Wen-Yu, Zhang Tong, Zhang Dong-Yan , Li Cai-Hong ,Wen Jian-Ping , Du Lian-Xiang, A novel bioflocculant produced by Enterobacter aerogenes and its use in defecating the trona suspension. Biochem. Eng. J., 27, pp. 1-7, 2005.

[13] Zheng Yan, Ye Zhi-Long, Fang Xu-Liang, Li Ya-Hong, Cai Wei-Min, Production and characteristics of a bioflocculant produced by Bacillus sp. F19. Bioresour. Technol., 99, pp.76867691, 2008.

[14] Ahmad, H. R. A.; Azni, I.; Norhafizah, A.; Rosfarizan M., Production and characterization of a bioflocculant produced by Aspergillus flavus. Bioresour. Technol., pp. 127, pp. 489-493, 2013.

[15] Li Yin; He Ning; Guan H.; Du G.; Chen J., A novel polygalacturonic acid bioflocculant REA11 produced by Corynebacterium glutamicum:a proposed biosynthetic pathway and experimental confirmation. Appl. Microbiol. Biotechnol., 63, pp. 200-206, 2003.

[16] Ma Fang, Liu Jun-Liang, Li Shu-Geng, Yang Ji-Xian, Zhang Li-Qiu,Wu Bo, Zhu Yan-Bin, Development of complex microbial flocculant. China Water \& Wastewater, 19, pp.1-4, 2003.

[17] Fujita, M.; Ike, M.; Tachibana, S.; Kitada, G.; Kim, S.M.; Inoue, Z., Characterization of a bioflocculant produced by Citrobacter sp. TKF04 from acetic and propionic acids. J. Biosci. Bioeng., 89, pp.40-46, 2000.

[18] Ma Fang, Zhu Yan-bin, Ren Nan-Qi, Yang Ji-xian, Compound bioflocculant, China Patent, ZL 200610010369.6, 2007.

[19] Wang Li-Li, Ma Fang, Qu Yuan-Yuan, Sun De-Zhi, Li Ang, Guo Jing-Bo, Bing Yu, Characterization of a compound bioflocculant produced by mixed culture of Rhizobium radiobacter F2 and Bacillus sphaeicus F6. World J. Microbiol. Biotechnol., 27, pp.2559-2565, 2011.

[20] Zhu Yan-bin, Feng Min, Yang Ji-Xian, Ma Fang, Wu Bo, Li Shu-Geng, Huang Jun-Li, Screening of complex bioflocculant producing bacteria and their flocculating mechanism, Journal of Harbin Institute of Technology, 36, pp.759-762, 2004.

[21] Li Jian, Wang Shu-Guang, Gao Bao-Yu,Yue Qin-Yan,Feng Gui-Ying, Study of a bioflocculant by dairy wastewater and its application, Environmental Engineering, 22, pp.93-94, 2004.

[22] Zhou Xu, Wang Jing, Zhou Ji-Ti, Zhang Jin-Song, Studies on properties of a bioflocculant produced by Pseudomonas sp. GX4-1 in fish meal wastewater, Research of Environmental Sciences, 16, pp.31-34,2003. 
[23] Ma Fang, Zhang Hui-Wen, Li Da-Peng, Wei Li, Hou Ning, Compound bioflocculant produced by using rice straw as substrate, China Environmental Science, 29, pp.196-200, 2009.

[24] Zhang Fan, Jiang Wen-Ju, Wang Xiang-Dong; Huang Juan; Xiao Jun, Wang Yi-Na; Zhang Wang, Chen Jiao, A new complex bioflocculant produced by mix-strains in alcohol wastewater and the characteristics of the bioflocculant, Fresenius Environmental Bulletin, 20, pp.2238-2245, 2011.

[25] Lu Yan, Qiao Fu-Zhen, Meng Li-Li, Study on the culture of complex bioflocculant producing bacterium in brewery wastewater, Technology of Water Treatment, 36, pp.31-33, 2010.

[26] Ren Hong-Yang, Wang Xin-Hui, Liu Da-Yu, Production and properties of bioflocculant by Aspergillus sojae and Pichia membranifaciens cultured in soy sauce waster,China Environmental Science, 30, pp.1050-1055, 2010.

[27] Li Da-Peng, Ma Fang, Hou Ning, Zhang Hui-Wen, Zhu Yan-Bin, Ke Bao-Qing, Preparation of compound bioflocculants by using waste zymotic fluid of glutamic acid fermentation as substrate, Journal of Hunan University(Natural Sciences), 36, pp.78-82, 2009.

[28] Ma Fang, Feng Min, Li Shu-Geng,Yang Ji-Xian, Research for flocculation effect of combined microbial flocculation agent, Journal of Heilongjiang Institute of Science \& Technology, 14, pp.140-144, 2004.

[29] Wang Qin, Wang Hui, Ma Fang, Zhang Jin-Feng, Yang Ji-Xian, Research on application of compound bioflocculant, Industrial Water Treatment, 27, pp. 68-71, 2007.

[30].Zhang Yu-Ling, Zhang Lan-Ying,Yao Jun, Fang Fang, Gao Sheng, High-efficient compound microbial flocculant, Journal of Harbin Institute of Technology, 40, pp.1481-1484, 2008.

[31] Ji Xiu-Juan,Jiang Wen-Ji,Zhang Fan,Wang Xiang-dong, Penformance and application of compound microbial flocculant XJBF-1, China Water \& Wastewater, 26, pp.74-76, 2010. 